\title{
Timing of reflexive visuospatial orienting in young, young-old, and old-old adults
}

\author{
Linda K. Langley • Chris Kelland Friesen • \\ Alyson L. Saville • Annie T. Ciernia
}

Published online: 11 March 2011

(C) Psychonomic Society, Inc. 2011

\begin{abstract}
This study examined adult age differences in reflexive orienting to two types of uninformative spatial cues: central arrows and peripheral onsets. In two experiments using a Posner cuing task, young adults (ages $18-28$ years), young-old adults (60-74 years), and old-old adults (75-92 years) responded to targets that were preceded $100-1,000 \mathrm{~ms}$ earlier by a central arrow or a peripheral abrupt onset. In Experiment 1, the cue remained present upon target onset. Facilitation effects at short cuetarget stimulus onset asynchronies (SOAs) were prolonged in duration for the two older groups relative to the young adults. At longer cue-target SOAs, inhibition of return (IOR) that was initiated by peripheral onset cues was observed in the performance of young adults but not in that of the two older groups. In Experiment 2, the cue was presented briefly and removed prior to target onset. The change in cue duration minimized age differences (particularly for young-old adults) in facilitation effects and led to IOR for all three age groups. The findings are consistent with the idea that attentional control settings change with age, with higher settings for older adults leading to delayed disengagement from spatial cues.
\end{abstract}

Keywords Aging · Attention - Spatial orienting · Young-old and old-old $\cdot$ Attentional control settings

Responding quickly to events in a complex visual world often requires rapid shifts of spatial attention. An attentional shift is

L. K. Langley $(\triangle) \cdot$ C. K. Friesen $\cdot$ A. L. Saville $\cdot$ A. T. Ciernia Department of Psychology and the Center for Visual and Cognitive Neuroscience, North Dakota State University, Fargo, ND 58108-6050, USA

e-mail: linda.langley@ndsu.edu

C. K. Friesen

e-mail: chris.friesen@ndsu.edu reflexive when it cannot be prevented, even when the external stimulus that elicits the shift does not provide information regarding the likely location of a desired item. It was originally thought that reflexive orienting occurred only toward stimuli that appeared or changed at a location peripheral to an observer's direct line of sight. Reflexive orienting is now known to also occur for certain uninformative central stimuli (e.g., Ristic, Friesen, \& Kingstone, 2002). In this study, we examined age-related changes in the time course of reflexive spatial orienting to both central arrows and peripheral onset cues. It has been proposed that with age, attentional control settings that regulate reflexive orienting are altered (Klein, 2000). We examined how these changes affect the time course with which older adults reflexively orient to uninformative peripheral and central visual information.

\section{Volitional versus reflexive orienting}

In the Posner cuing paradigm (Posner \& Cohen, 1984), which has been widely used to study spatial orienting, a central fixation point is flanked by visual markers (e.g., two boxes) that indicate potential target locations. One of two types of cues has traditionally been presented: an arrow cue at central fixation that points to one of the potential target locations, or a sudden-onset cue at one of the peripheral locations (e.g., a brief highlighting of the outline of one of the boxes). A target is presented shortly after the cue, to which participants make a speeded detection (e.g., press a button as soon as the target is detected) or discrimination (e.g., press a button to indicate the location of the target) response. In early research, central arrows were informative (the target more often appeared at the location indicated by the arrow than at an uncued location) and peripheral cues 
were uninformative (the target was just as likely to appear at an uncued location as at the cued location). Both types of cues were effective in orienting attention. Observers were faster to respond to targets that were presented at cued locations (validly cued targets) than to those presented at uncued locations (invalidly cued targets). This facilitation effect was attributed to a "spotlight" of attention (Posner \& Cohen, 1984). The cue caused the spotlight to be directed toward the cued location, and once attention was engaged there, a target was detected efficiently if it fell under the attentional spotlight. If the target was presented at an uncued location, responses were slowed because attention had to be disengaged from the cued location and the spotlight had to be moved to the target location.

Temporal patterns of orienting were found to vary for the two types of cue (for a review, see Klein, Kingstone, \& Pontefract, 1992). With informative central arrow cues, facilitation effects were maximal at approximately $300 \mathrm{~ms}$ and were maintained at longer intervals. In contrast, facilitation effects developed rapidly with uninformative peripheral cues (as early as 50-100 ms), but they were replaced by inhibitory effects at approximately $300 \mathrm{~ms}$, with slower responses to validly than to invalidly cued targets. The contrast in cuing patterns was interpreted as reflecting volitional orienting in response to informative central cues and reflexive orienting toward uninformative peripheral cues (Jonides, 1981). With central arrows, observers voluntarily directed their attention to the cued location based on their knowledge that the arrows were predictive of the target's likely location. Because observers expected the target to appear at the cued location, attention was not quickly withdrawn from cued locations, leading to prolonged facilitation effects. Peripheral cues were thought to summon attention automatically (i.e., attention was reflexively drawn to stimuli moving or appearing in the periphery), leading to facilitation effects at short cue-target intervals. At longer intervals, when the target did not immediately appear at the cued location, attention returned to central fixation because there was no expectation that the target would appear at the cued location. If the target subsequently did appear at the cued location, attention was slower to return because of a bias to explore new locations and to avoid reinspecting previous locations. The resulting slowing in reaction times (RTs) was termed inhibition of return (IOR; Posner \& Cohen, 1984; see Klein, 2000, for a review).

Contrary to early assumptions (e.g., Jonides, 1981), spatial orienting that is guided by central arrow cues can be reflexive as well as voluntary. It has recently been demonstrated that even when an arrow cue is not informative, it can facilitate target detection (e.g., Hommel, Pratt, Colzato, \& Godijn, 2001; Kingstone, Smilek, Ristic, Friesen, \& Eastwood, 2003; Ristic et al., 2002; Tipples, 2002). Like the orienting pattern identified with peripheral cues, facilitation effects resulting from uninformative central arrows have been observed at short cue-target intervals. In contrast to peripheral cues, inhibitory effects are not observed at longer intervals, consistent with the idea that IOR is linked to the eye movement system, which is activated by a visual transient in the periphery. Because these orienting effects arise early and occur despite the fact that the arrows are not predictive of the likely target location, they can be viewed as reflexive or automatic. Thus, the orienting effects observed with the informative arrows, which had been interpreted to reflect volitional orienting, likely represented some combination of reflexive and volitional orienting (Ristic \& Kingstone, 2006).

\section{Adult age differences in visuospatial orienting}

Arrow cues Age differences in orienting to arrow cues have been studied with informative arrows only. Older adults' attention is effectively guided by arrows that predict the likely location of a target. In fact, facilitation effects for older adults are similar in magnitude to those for young adults (Curran, Hills, Patterson, \& Strauss, 2001; Tales, Muir, Bayer, \& Snowden, 2002; Tellinghuisen, Zimba, \& Robin, 1996; Yamaguchi, Tsuchiya, \& Kobayashi, 1995), or are even greater in magnitude (Folk \& Hoyer, 1992; Hartley, Kieley, \& Slabach, 1990; Lincourt, Folk, \& Hoyer, 1997; Nissen \& Corkin, 1985). Greater facilitation effects have been attributed to either general slowing on the part of older adults (Lincourt et al., 1997) or to age-related difficulties in cue encoding (Folk \& Hoyer, 1992). In addition, the time courses of facilitation effects tend to be similar for the two age groups (Folk \& Hoyer, 1992; Lincourt et al., 1997; Yamaguchi et al., 1995). Typically, facilitation effects are minimal at cue-target intervals of $50 \mathrm{~ms}$ or less but are clearly evident at stimulus onset asynchronies (SOAs) of $250 \mathrm{~ms}$ and longer (up to $3,000 \mathrm{~ms}$ ), with no indication of IOR at longer intervals. In some cases, facilitation effects have emerged earlier for older adults, relative to young adults (e.g., Hartley et al., 1990), and in other cases they have emerged later (e.g., Brodeur \& Enns, 1997).

Peripheral cues The timing of reflexive orienting to uninformative peripheral cues changes with age. Relative to young adults, older adults tend to show extended and enhanced cue facilitation at short cue-target intervals (e.g., 50-200 ms; Brodeur \& Enns, 1997; Castel, Chasteen, Scialfa, \& Pratt, 2003; Lincourt et al., 1997) and delayed IOR at longer intervals (Brodeur \& Enns, 1997; Castel et al., 2003), which may be due to older adults having greater difficulty disengaging attention from cues (Castel et al., 2003). When peripheral cues are informative (predictive or counterpredictive), the cuing effects for young and older adults are consistent with rapid and automatic orienting at 
short cue-target intervals (facilitated responding to cued locations, even when the cue predicts that the target will appear elsewhere; Faust \& Balota, 1997; Hartley \& Kieley, 1995; Hartley et al., 1990; Yamaguchi et al., 1995).

\section{Visuospatial orienting for young-old and old-old adults}

Orienting processes continue to change late in life, at least when the task is sufficiently challenging (Greenwood \& Parasuraman, 1994; Greenwood, Parasuraman, \& Haxby, 1993). With a simple target detection task, adults across a wide age range (17-85 years) show similar orienting patterns in response to informative arrow cues (Greenwood \& Parasuraman, 1994; Greenwood et al., 1993). However, when completing a letter discrimination task, facilitation effects for old-old adults (ages 75-84 years) are greater than those for young-old adults (ages 65-74 years). The age difference appears to be due largely to slower disengagement from an invalid cue rather than faster engagement of a valid cue. Orienting to noninformative cues has not yet been investigated in old-old adults.

\section{Attentional control settings}

To summarize, both young adults and older adults orient in response to peripheral onset cues and arrow cues. When orienting to peripheral onset cues, older adults tend to show greater facilitation than young adults do at early cue-target intervals. Furthermore, older adults maintain facilitation longer and demonstrate a delayed onset of IOR relative to young adults. Volitional orienting to informative central arrows is unchanged with age, or if anything, older adults are more sensitive to cue information than are young adults (as demonstrated by greater facilitation effects). Further age enhancements in facilitation effects are observed for adults over the age of 75 , but only when the task is sufficiently difficult. However, reflexive orienting to noninformative central arrows has yet to be studied in older adults.

To interpret the pattern of findings described above, we turn to the attentional control setting (ACS) theory (Folk, Remington, \& Johnston, 1992). According to this theory, when a task is more difficult, people allocate more attentional resources for longer periods of time than when the task is simpler. Specifically, the observer sets the level of attention to be allocated to a target based on task difficulty (i.e., low intensity for a simple task, high intensity for a difficult task). The attentional control settings cannot be changed fluidly, so the level of attention selected to process the target will also apply to the cue. The higher the control setting, the more strongly attention is directed to a cue, leading to extended facilitation and (in the case of peripheral cues) later emergence of IOR. Klein (2000) proposed that the ACS theory could account for task differences and individual differences in the time course of attentional orienting. For example, the theory can account for the later emergence of IOR on discrimination tasks relative to detection tasks (Klein, 2000; Lupiáñez, Milliken, Solano, Weaver, \& Tipper, 2001), because greater attention is required for discrimination than for detection. It is also consistent with findings that individuals with poorer attentional control, such as young children (MacPherson, Klein, \& Moore, 2003) and individuals with schizophrenia (Huey \& Wexler, 1994; Sapir, Henik, Dobrusin, \& Hochman, 2001; Spencer et al., 2011), show enhanced facilitation and delayed IOR relative to their respective comparison groups (older children and adult controls).

Older adults, like young children, might apply different attentional control settings to spatial-orienting tasks (Klein, 2005). Because the target-processing task becomes more difficult with age, older adults may set their control settings at a higher level than young adults do. With greater attention to the cue, older adults would be slower to disengage from the cued location, and thus would demonstrate greater and longer-lasting benefits of a valid cue and, with uninformative peripheral cues, later onset of IOR. The age pattern predicted by ACS theory is consistent with the orienting patterns of older adults summarized above.

Does the ACS theory make similar predictions for the orienting patterns resulting from central cues and from peripheral cues? The answer depends on whether the control settings affect engagement and disengagement with the cue itself or with the location indicated by the cue. If a higher setting leads to greater engagement with the cue, it would seem that engagement of the central arrow would lead to reduced orienting to the cued location. However, if the result of a higher control setting is greater attention to the location indicated by the cue (an enhanced orienting effect), facilitation effects would then be enhanced. We propose that attentional control settings impact how strongly observers react to the directional information provided by the cue rather than how long observers' attention dwells on the cue, which leads to the prediction that age-related increases in facilitation effects would be observed for both central arrows and peripheral onset cues.

\section{The present study}

The purpose of the present study was to examine age differences in reflexive orienting to uninformative peripheral onset cues and uninformative central arrow cues. We assessed orienting performance in young adults (ages 18-30 years), 
young-old adults (60-74 years), and old-old adults ( $75+$ years). If the ACS theory can account for age differences in reflexive orienting, we would expect that both groups of older adults would show facilitation effects of greater magnitude and longer duration than would younger adults, for both peripheral and central cues. If old-old adults find the target task (location discrimination) particularly difficult, they should implement an even higher control setting than do young-old adults, leading this group to have even stronger and longer-lasting facilitation effects. For peripheral cues, the two older groups should demonstrate a later onset of IOR as compared to young adults, due to delayed disengagement from the cue. We used a twolocation Posner cuing task with a variable cue-target SOA and a location discrimination response. To examine the impact of cue duration on age differences in reflexive orienting, the cue remained on the screen during target presentation in Experiment 1 and was removed prior to target presentation in Experiment 2. If age was associated with difficulty in cue disengagement, age effects were expected to be accentuated for persistent cues.

\section{Experiment 1}

The purpose of Experiment 1 was to determine whether the reflexive orienting patterns for young adults, young-old adults, and old-old adults would be consistent with the age differences predicted by ACS theory. We expected that both peripheral onset cues and central arrow cues would lead to facilitation effects at a cue-target SOA of $100 \mathrm{~ms}$, consistent with reflexive orienting (e.g., Hommel et al., 2001; Klein, 2000; Posner \& Cohen, 1984; Ristic et al., 2002; Tipples, 2002). At longer intervals (SOAs of 600 and $1,000 \mathrm{~ms}$ ), facilitation effects would diminish for central arrow cues because the arrows would not predict target location (Ristic et al., 2002). For peripheral onset cues, early facilitation would turn into IOR, biasing attention to new locations (Klein, 2000; Posner \& Cohen, 1984). Because the location discrimination decision required of the target would be more difficult for older than for young adults, we predicted that the orienting performance of the two older groups (young-old and old-old) would reflect greater and longer-lasting facilitation effects (for both peripheral and central cues) and delayed onset of inhibitory effects (for peripheral cues), as the result of a higher attentional control setting that would lead to enhanced processing of cue information.

\section{Method}

Participants Thirty-four young adults (18-28 years; 22 women, 12 men), 34 young-old adults (61-73 years; 22 women, 12 men), and 34 old-old adults (75-92 years; 22 women, 12 men) participated in Experiment 1. Young adults were recruited from psychology courses and received course credit. Older adults were recruited from the Fargo-Moorhead community and received $\$ 10 / \mathrm{h}$ for participating. All individuals had at least a high school education and were native English speakers. Participants had corrected near visual acuity of 20/40 or better, as assessed by a Snellen eye chart (Precision Vision, La Salle, IL), and were free from serious medical conditions (e.g., cancer, stroke, dementia, or drug and alcohol abuse) according to self-report (Christensen, Moye, Armson, \& Kern, 1992). All of the included participants scored 9 points or lower on the Geriatric Depression Scale (GDS; Yesavage et al., 1982), indicating minimal depressive symptoms, and 26 points or higher on the Mini-Mental State Examination (MMSE; Folstein, Folstein, \& McHugh, 1975), indicating no demonstrable signs of significant cognitive impairment. Demographic and screening data for the included participants are provided in Table 1.

Materials and stimuli The stimuli were presented on a 17 -in. color monitor (refresh rate of $85 \mathrm{~Hz}$ ) controlled by a PC computer with a Pentium 4 processor. A chinrest maintained participants' viewing distance at $40 \mathrm{~cm}$. Participants responded to stimuli using a PST Serial Response Box (Psychology Software Tools, Pittsburgh, PA). We used E-Prime 1.1 (Psychology Software Tools) to develop and run the experiment. The stimuli were black line drawings presented against a white background. The initial fixation display consisted of a circle positioned in the middle of the monitor that subtended $14.5^{\circ}$ of visual angle. Centered in the circle was a cross with arms that were $4.3^{\circ}$ long and $0.1^{\circ}$ thick. The circle was flanked to the left and right by two empty $2.9^{\circ}$ squares. The outer edges of the squares were located $8.9^{\circ}$ from the outer edge of the central circle. Sample stimulus displays are presented in Fig. 1. The cue displays (arrow and peripheral cues) overlaid the fixation display. The arrow was asymmetrical, with a shaft (4.3 $\mathrm{long})$ and head $\left(1.4^{\circ}\right.$ high and $1.0^{\circ}$ wide) pointing to the left or right, drawn with lines $0.4^{\circ}$ thick. The arrow overlaid the horizontal line of the center fixation cross. The peripheral cue consisted of an empty square that superimposed one of the two outer squares. The outline of the square was thicker than in the original display $\left(0.6^{\circ}\right.$ instead of approximately $\left.0.1^{\circ}\right)$. A black filled circle, $1.7^{\circ}$ in diameter, served as the target stimulus for both cue conditions and was presented in the center of one of the two outer squares.

Design and procedure The testing session (including consent, screening, and computer task) lasted approximately $1 \frac{1}{2}$ hours. The computer task consisted of two blocks of 80 trials for each cue type (arrow and peripheral), for a total of 320 experimental trials. Blocks alternated between arrow and peripheral cues 
Table 1 Participant characteristics for Experiments 1 and 2

\begin{tabular}{|c|c|c|c|c|c|c|c|c|c|c|c|c|}
\hline & \multicolumn{6}{|c|}{ Experiment 1} & \multicolumn{6}{|c|}{ Experiment 2} \\
\hline & \multicolumn{3}{|l|}{ Mean } & \multicolumn{3}{|l|}{ SD } & \multicolumn{3}{|l|}{ Mean } & \multicolumn{3}{|l|}{$\mathrm{SD}$} \\
\hline & YA & YO & $\mathrm{OO}$ & YA & $\mathrm{YO}$ & $\mathrm{OO}$ & YA & YO & $\mathrm{OO}$ & YA & YO & $\mathrm{OO}$ \\
\hline Age (yrs) & $20.7^{\mathrm{a}}$ & 67.0 & $79.3^{\mathrm{a}}$ & 2.4 & 3.7 & 3.8 & $20.3^{\mathrm{a}}$ & 67.3 & $79.2^{\mathrm{a}}$ & 2.4 & 4.1 & 3.0 \\
\hline Education (yrs) & 14.1 & 15.5 & 15.1 & 1.4 & 3.3 & 2.8 & 14.0 & 14.9 & 14.8 & 1.3 & 2.4 & 2.4 \\
\hline GDS (30 max) & 2.2 & 1.3 & 1.4 & 2.4 & 1.4 & 2.2 & 1.5 & 1.7 & 2.1 & 2.1 & 2.3 & 2.0 \\
\hline WASI Vocab. (80 max) & $58.8^{\mathrm{a}}$ & 68.0 & 66.3 & 6.4 & 7.5 & 9.0 & $58.5^{\mathrm{a}}$ & 65.0 & 64.0 & 5.4 & 8.0 & 6.5 \\
\hline Snellen acuity $(20 / \ldots)$ & $16.1^{\mathrm{a}}$ & 22.8 & 24.9 & 4.4 & 6.7 & 6.6 & $15.9^{\mathrm{a}}$ & 24.7 & 25.8 & 4.1 & 6.6 & 7.0 \\
\hline MMSE (30 max) & 29.0 & 29.4 & 29.1 & 1.0 & 0.9 & 0.8 & 29.6 & 29.4 & 29.1 & 0.7 & 1.0 & 1.0 \\
\hline
\end{tabular}

YA, young adults; YO, young-old adults; OO, old-old adults. GDS, Geriatric Depression Scale: Maximum score is 30, with a higher score indicating more endorsed symptoms of depression. WASI, Wechsler Abbreviated Scale of Intelligence (Wechsler, 1999): Maximum score on the Vocabulary subscale is 80 points, with a higher score indicating better performance. For Snellen acuity, a smaller denominator of the Snellen fraction for corrected near vision indicates better vision. MMSE, Mini Mental State Examination: Maximum score is 30 points, with a higher score indicating better performance. ${ }^{a}$ Mean scores differed significantly from the young-old adult group according to a Student/Newman-Keuls $t$ test, $p<.05$

( $\mathrm{ABAB}$ or $\mathrm{BABA})$; whether an arrow or peripheral block was presented first was counterbalanced across participants. Before each block, participants completed eight practice trials that were the same in format as the experimental trials.

As depicted in Fig. 1, a trial began with the fixation display presented for $995 \mathrm{~ms}$, followed by a cue display
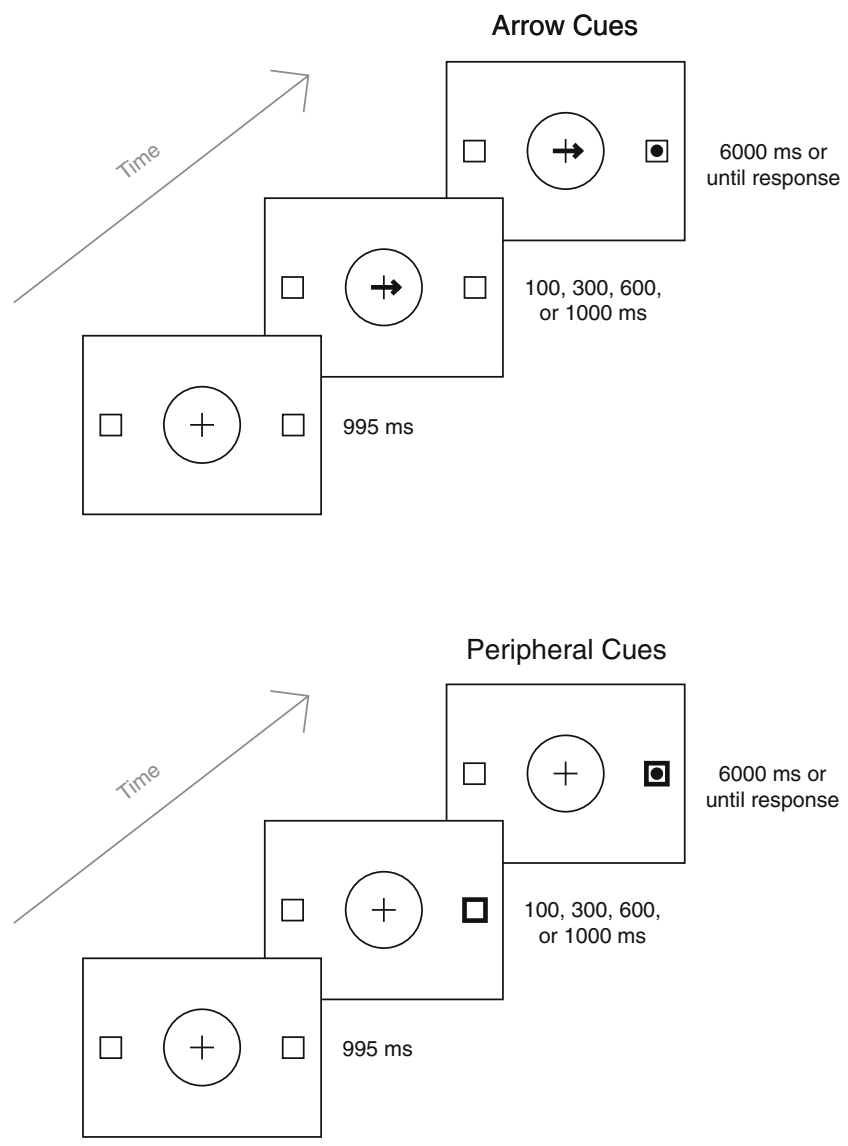

Fig. 1 Trial sequence for Experiment 1. The stimuli are not scaled to size (an arrow pointing left or right, or a thick left or right square outline). After a variable cue-target SOA (specific times are described below), the target appeared in either the left or the right outer square. The cue and target remained on the screen for $6,000 \mathrm{~ms}$ or until the participant responded. Participants pressed the left or right buttons specified on the response box to indicate the target's location.

In addition to cue type (arrow or peripheral cue), the variables of interest were cue validity and cue-target SOA. On valid trials, the target appeared at the location indicated by the cue (left or right), whereas on invalid trials the target appeared at the uncued location. The SOAs were four values close to $100,300,600$, and $1,000 \mathrm{~ms}$ (as constrained by the refresh rate of the monitor): 117.6, 317.6, 611.7, and $1,011.7 \mathrm{~ms}$. For convenience, we will refer to these SOAs as $100,300,600$, and $1,000 \mathrm{~ms}$, respectively, throughout the remainder of this report. All combinations of cue direction/side, target side, and SOA were selected randomly and with equal probability within each block.

The experimenter explained the task to participants using verbal instructions and a drawn representation of the stimulus events. Participants were told that the direction or location of the cue (left or right) was random and would not help them predict the target location. They were instructed to rest their index fingers on two buttons of the response box and to indicate the target location by pressing the left or the right button as quickly as possible, but not at the expense of accuracy. Participants took short rests between blocks.

\section{Results}

Mean RTs as a function of cue type, age group, cue validity, and cue-target SOA are presented in Table 2. Responses 
Table 2 Mean reaction times in milliseconds (and standard deviations) for Experiment 1

\begin{tabular}{|c|c|c|c|c|c|c|c|c|}
\hline \multirow[b]{2}{*}{ SOA (ms) } & \multicolumn{4}{|c|}{ Arrow Cues } & \multicolumn{4}{|c|}{ Peripheral Cues } \\
\hline & 100 & 300 & 600 & 1,000 & 100 & 300 & 600 & 1,000 \\
\hline \multicolumn{9}{|l|}{ Young } \\
\hline Valid & $383(67)$ & $342(59)$ & $335(65)$ & $328(57)$ & $395(68)$ & $368(55)$ & $366(64)$ & $352(65)$ \\
\hline Invalid & $399(68)$ & $348(63)$ & $331(62)$ & $339(70)$ & $415(66)$ & $374(76)$ & $350(75)$ & $343(60)$ \\
\hline \multicolumn{9}{|l|}{ Young-old } \\
\hline Valid & 474 (76) & $437(65)$ & 404 (59) & $416(65)$ & $486(68)$ & $449(63)$ & $436(61)$ & 439 (69) \\
\hline Invalid & 499 (74) & $464(67)$ & $416(67)$ & $420(71)$ & $531(71)$ & $480(73)$ & $446(74)$ & 438 (78) \\
\hline \multicolumn{9}{|l|}{ Old-old } \\
\hline Valid & $523(81)$ & $483(85)$ & $453(76)$ & $463(96)$ & $544(80)$ & $499(82)$ & $492(87)$ & $500(94)$ \\
\hline Invalid & $559(92)$ & $521(87)$ & $460(97)$ & 455 (94) & $597(95)$ & $534(100)$ & 499 (107) & 496 (108) \\
\hline
\end{tabular}

that were less than $150 \mathrm{~ms}$ or more than 2,000 ms in latency were considered outliers and removed. For the remaining trials, median RTs were calculated for correct responses. We conducted a $3 \times 2 \times 4$ mixed ANOVA for each cue type, with age group (young, young-old, and old-old adults) as the between-subjects factor, and cue validity (valid and invalid) and cue-target SOA $(100,300,600$, and 1,000 ms) as within-subject factors. For all analyses, we used an alpha of .05 , and for simple effects analyses of variables with more than two levels we used Student/Newman-Keuls (SNK) post-hoc tests to reduce experiment-wise error rates.

Arrow cues For arrow cues, all three main effects were significant: age group, $F(2,99)=34.48, p<.0001$, cue validity, $F(1,99)=49.60, p<.0001$, and cue-target SOA, $F(3,297)=230.41, p<.0001$. Old-old adults were slower to respond than young-old adults, who in turn were slower to respond than young adults $(490,441$, and $351 \mathrm{~ms}$, respectively), $p \mathrm{~s}<.05$. The main effect for cue validity indicated a significant cue facilitation effect; overall, participants responded more quickly to validly cued targets (420 ms) than to invalidly cued targets (434 ms). The main effect of SOA reflected a general decrease in RT as SOA increased (473, 433, 400, and $404 \mathrm{~ms}$ for SOAs of 100, 300,600 , and 1,000 ms, respectively), a standard foreperiod effect (e.g., Friesen \& Kingstone, 1998).

In addition to the main effects, there was a significant two-way interaction between cue validity and SOA (consistent with declining facilitation effects with increasing SOA), $F(3,297)=16.59, p<.0001$, which was qualified by a three-way interaction between age group, cue validity, and $\mathrm{SOA}, F(6,297)=4.59, p<.001$. We explored the three-way interaction with simple main effects testing to examine validity effects for each age group at each SOA. The two older groups' RTs were significantly faster following valid cues than following invalid cues at the $100-$ and 300-ms SOAs, all $F_{\mathrm{s}}>17.0$, all $p \mathrm{~s}<.001$. In contrast, young adults' RTs reflected this pattern only at the 100-ms SOA, $F(1,33)=13.13, p<.001$. Consistent with this pattern, when difference scores (invalid RT minus valid RT) were submitted to one-way ANOVAs to assess group differences in validity effects at each of the SOAs, there was a significant age effect at the 300-ms SOA, $F(2,99)=8.22$, $p<.001$, with the two older groups having significantly greater facilitation effects than the young group, as indicated by SNK post-hoc comparisons, $p \mathrm{~s}<.05$. Difference scores reflecting facilitation effects for arrow cues are depicted in Fig. 2a.

Given the significant effects of age in the analysis above, we conducted a second set of analyses on transformed RTs to reduce the influence of general slowing on group differences in facilitation effects (Madden, Pierce, \& Allen, 1992; Madden, Whiting, Cabeza, \& Huettel, 2004; see also Faust, Balota, Spieler, \& Ferraro, 1999, for a similar approach). We used Brinley plot analyses (Cerella, 1994) of the eight condition means to determine the regression equations that best characterized the linear relationship between the means of old-old adults and the two other groups. We used the resulting equations (see Eqs. 1 and 2 below) to transform the data of young and young-old participants. The assumption of this approach was that age interactions that remained significant following the transformation could be considered representative of cognitive or perceptual effects that were independent of general slowing. Submitting the transformed data to the $3 \times 2 \times 4$ ANOVA described above, there was no longer a main effect of age, $F<1$, as expected. However, the main effects of cue validity, $F(1,99)=50.00, p<.0001$, and SOA, $F(3,297)=239.12, p<.0001$, remained significant, as did the three-way interaction of age group, cue validity, and SOA, $F(6,297)=3.70, p<.01$. As in the original analysis, the cue validity effects of the two older groups were significantly greater than those of young adults at the 300 -ms SOA, $F(2,99)=6.11, p<.01$.

Old-Old RT $=1.38($ Young RT $)+4, r^{2}=.82$

Old-Old RT $=1.16($ Young-Old RT $)-23, r^{2}=.98$ 


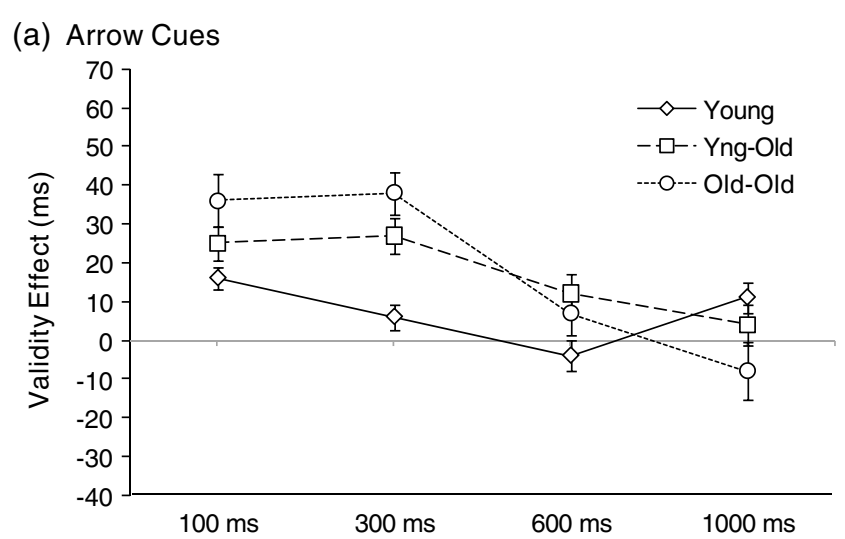

(b) Peripheral Cues

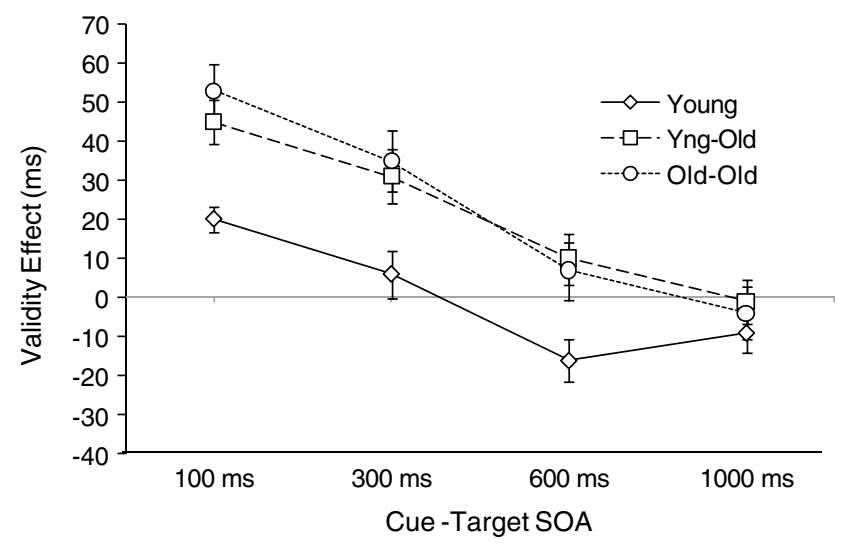

Fig. 2 Validity effects (difference scores: invalid RT minus valid RT) as a function of cue-target SOA for young, young-old, and old-old adults in Experiment 1. a Uninformative central arrow cues. b Uninformative peripheral onset cues. Error bars represent \pm 1 SEM

Peripheral cues Replicating the pattern found with arrow cues, the three main effects of the $3 \times 2 \times 4$ ANOVA were significant: age group (old-old adults were significantly slower than young-old adults, who in turn were slower than young adults), $F(2,99)=37.05, p<.0001$; cue validity (participants responded more quickly to validly than to invalidly cued targets), $F(1,99)=26.16$, $p<.0001$; and cue-target SOA (participants responded more quickly to targets presented at longer SOAs), $F(3,297)=178.41, p<.0001$. In addition, there were two significant two-way interactions: Age Group $\times$ Cue Validity, $F(2,99)=6.62, p<.01$, and Cue Validity $\times$ SOA, $F(3,297)=36.32, p<.0001$. The three-way interaction was not significant, $F(6,297)=1.23, p>.20$. We explored the Age Group $\times$ Cue Validity interaction by conducting one-way ANOVAs of validity effects for each age group, collapsed across SOAs. Responses were significantly faster to validly than to invalidly cued targets for old-old adults (by $23 \mathrm{~ms}$ ), $F(1,33)=13.50$, $p<.001$, and for young-old adults (by $21 \mathrm{~ms}$ ), $F(1,33)=$ $18.67, p<.0001$, but not for young adults $(0 \mathrm{~ms}), F<1$. We explored the Cue Validity $\times$ SOA interaction by conducting within-subject ANOVAs on the validity effects for each SOA. There were significant facilitation effects at the $100-\mathrm{ms}$ SOA $(39 \mathrm{~ms}), F(1,101)=74.99$, $p<.0001$, and at the $300-\mathrm{ms} \mathrm{SOA}(24 \mathrm{~ms}), F(1,101)=$ $35.64, p<.0001$. Contrary to predictions, there were not significant IOR effects at the $600-$ or $1,000-\mathrm{ms}$ SOAs ( 1 and $-4 \mathrm{~ms}$, respectively).

To be consistent with the analysis for arrow cues, we examined each age group's validity effects at each SOA with simple main effects testing. The performance of the two older groups reflected significant facilitation effects at the 100- and 300-ms SOAs, all $F \mathrm{~S}>27.0$, all $p$ s $<.0001$. Young adults demonstrated significant facilitation at the $100-\mathrm{ms}$ SOA, $F(1,33)=23.67, p<.0001$, and significant IOR effects at the 600- and 1,000-ms SOAs, both $F_{\mathrm{s}}>4.2$, both $p \mathrm{~s}<.05$. Group differences in validity effects, as assessed with difference scores (invalid RT minus valid RT), were significant at SOAs of $100 \mathrm{~ms}, F(2,99)=5.25$, $p<.01,300 \mathrm{~ms}, F(2,99)=5.85, p<.01$, and $600 \mathrm{~ms}$, $F(2,99)=3.89, p<.05$, with the two older groups demonstrating significantly greater facilitation (or at $600 \mathrm{~ms}$, significantly less inhibition) than the young adult group, $p<.05$. The validity-effect difference scores for peripheral cues are depicted in Fig. $2 b$.

The Brinley analyses on the peripheral cue data produced Eqs. 3 and 4, which we used to transform the data of young and young-old participants. When submitted to the overall ANOVA, the main effect of age was no longer significant, $F<1$, as expected. However, the main effects of cue validity, $F(1,99)=23.69, p<.0001$, and cue-target SOA, $F(3,297)=189.49, p<.0001$, continued to be significant, as did the interactions between age group and cue validity, $F(2,99)=5.97$, $p<.01$, and between cue validity and SOA, $F(3,297)=$ $35.34, p<.0001$. All remaining significance patterns remained unchanged from the original analysis, except that the group difference in validity effects at the $100-\mathrm{ms}$ SOA was now marginally significant, $F(2,99)=2.85$, $p=.06$.

Old-Old RT $=1.38($ Young RT $)+10, r^{2}=.84$

Old-Old RT $=1.08($ Young-Old RT $)+18, r^{2}=.99$

Errors For both arrow cues and peripheral cues, error rates were low across all conditions for each age group (ranging between $0 \%$ and $1.5 \%$ ), so no further error analyses were conducted. 


\section{Discussion}

Both central arrows and peripheral onsets were successful in rapidly orienting attention. At the shortest cue-target interval (100-ms SOA), participants were faster to localize targets preceded by valid cues than to localize those preceded by invalid cues. This early facilitation effect for uninformative arrows is consistent with recent proposals (Kingstone et al., 2003; Ristic \& Kingstone, 2006) that arrows represent a special form of central symbolic cue that has gained the ability to automatically direct spatial attention. Facilitation effects for arrows were no longer evident by $600 \mathrm{~ms}$, indicating that attention was not maintained at a cued location when the cue was not predictive (Ristic et al., 2002). Peripheral onset cues, in contrast, were associated with IOR (slower responses to validly than to invalidly cued targets) at longer SOAs (600 and 1,000 ms), consistent with a slowed return of attention to previously attended locations. This inhibitory effect, which was observed in the responses of young adults, is thought to be linked to oculomotor programming. For cues that trigger the preparation of an eye movement, such as stimuli peripheral to central gaze (Abrams \& Dobkin, 1994; Rafal, Calabresi, Brennan, \& Sciolto, 1989), attention is slower to return to cued locations. Thus, to summarize, the two types of spatial cues were associated with reflexive orienting patterns that differed in their time courses.

As was also predicted, orienting patterns differed by age. Facilitation effects were longer in duration for the two older adult groups relative to the young adult group. For arrow cues, young adults' facilitation effects were observed at $100 \mathrm{~ms}$ but had resolved by $300 \mathrm{~ms}$. Older adults' facilitation effects were still evident at $300 \mathrm{~ms}$, leading to significant age differences (which withstood an analysis accounting for general slowing) in the magnitude of facilitation effects at $300 \mathrm{~ms}$. For peripheral cues, the two older age groups showed greater facilitation than did young adults at the 100- and 300-ms SOAs (the age difference was still significant at the $300-\mathrm{ms}$ SOA after accounting for general slowing). Younger adults demonstrated IOR at the longer SOAs (600 and 1,000 ms), but the two older groups did not.

The present findings are consistent with the ACS theory (Klein, 2005). Prolonged facilitation effects for older adults suggested that they attended to cued locations with greater intensity than did young adults. According to the theory, this heightened sensitivity resulted because older adults perceived the target localization task to be more difficult, leading them to raise their attentional control settings relative to the setting used by young adults. The higher settings were applied to cues as well as to targets, which delayed the disengagement of attention from cued locations (leading to prolonged facilitation effects and delayed IOR). The old-old adults and young-old adults had similar orienting patterns, indicating that old-old adults did not need to further raise their ACS level to successfully perform the task.

Given the relatively long cue-target intervals (600- and 1,000-ms SOAs), we were surprised to find that IOR did not develop for the two older groups. Using a single-cue task similar to that used in the present study, Castel, Chasteen, Scialfa, and Pratt (2003) found that IOR was delayed with age but was still observed by approximately $600 \mathrm{~ms}$. However, an important difference between the two study designs is that in the present study, the cue remained on the screen during target presentation. If older adults were slow to disengage from the location indicated by the cue due to a high control setting, the continued presence of the cue may have exacerbated this delay in the onset of IOR. Indeed, leaving the cue on the screen throughout the experimental trial may have (1) enhanced age differences in facilitation effects at short intervals for both types of cues and (2) delayed the onset of older adults' IOR at longer intervals for peripheral cues. To assess this possibility, we removed the cue prior to target presentation in Experiment 2 to see whether age differences in initial orienting and IOR would diminish.

\section{Experiment 2}

In Experiment 2, we examined the impact of cue duration on age differences in the time course of orienting. Experiment 2 was identical to Experiment 1, except that the cue was presented for a fixed duration and was removed prior to target presentation. As observed in Experiment 1, we expected to find age differences in the magnitude and duration of cue validity effects. However, we predicted that removing the cue would facilitate endogenous disengagement from the cued location, particularly for older adults, and thus reduce age differences in facilitation effects. Furthermore, with earlier disengagement, IOR to peripherally cued locations would be more likely to be observed at the sampled SOAs, although IOR onset might still be delayed for older adults due to continued group differences in control settings.

\section{Method}

Participants Forty young adults (18-28 years; 27 women, 13 men), 40 young-old adults (60-74 years; 27 women, 13 men), and 40 old-old adults (75-86 years; 28 women, 12 men) participated in Experiment 2 (see Table 1 for the participants' screening and psychometric data). Participants were recruited and screened in the same manner as in Experiment 1, but there were no participants in common between the two experiments. 
Materials and procedure The stimuli, design, and procedure were the same as those described for Experiment 1, except that instead of overlapping temporally with the target, the cue was presented for $50 \mathrm{~ms}$ and was then removed. After an interstimulus interval of $50,250,550$, or $950 \mathrm{~ms}$ (corresponding to cue-target SOAs of 100, 300, 600, and $1,000 \mathrm{~ms}$ ), the target was presented until the participant responded or 6,000 ms had elapsed. Sample trial sequences are presented in Fig. 3. As in Experiment 1, participants were told that the direction or location of the cue was random and would not assist them in predicting the target location. Participants completed two blocks of 80 trials for each cue type (arrow and peripheral), for a total of 320 experimental trials. Each block began with 8 practice trials.

\section{Results}

Table 3 displays the mean RTs for Experiment 2 as a function of cue type, age group, cue validity, and cue-target SOA. After removing outliers and error trials, the median RTs were submitted to separate $3 \times 4 \times 2$ mixed ANOVAs for the two types of cues. We again used an alpha level of .05 and SNK post-hoc tests on variables involving more than two levels.

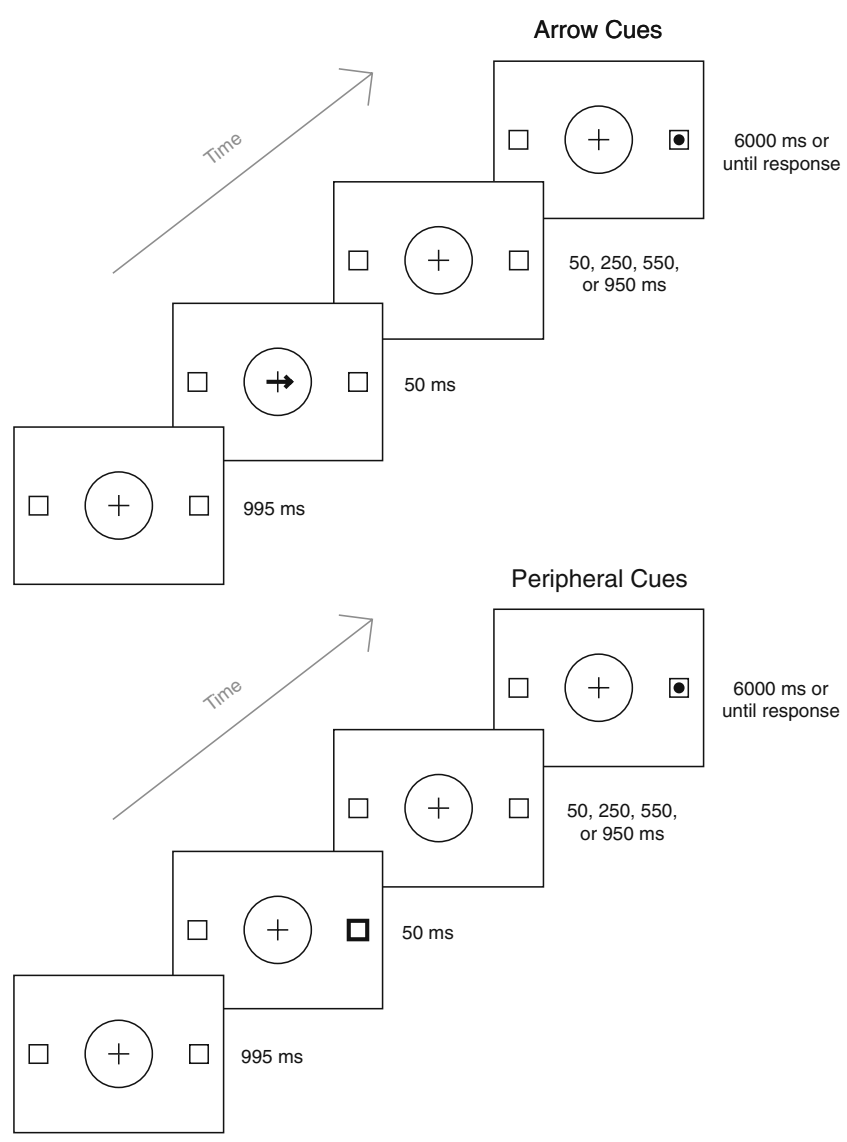

Fig. 3 Trial sequence for Experiment 2. The stimuli are not scaled to size
Arrow cues As in Experiment 1, the ANOVA for arrow cues revealed the three main effects to be significant: age group, $F(2,117)=46.05, p<.0001$, cue validity, $F(1,117)=35.43$, $p<.0001$, and cue-target SOA, $F(3,351)=200.05$, $p<.0001$. In addition, there was a significant two-way interaction between cue validity and SOA (reflecting declining facilitation effects with increasing $\mathrm{SOA}), F(3,351)=31.19$, $p<.0001$, which was qualified by a three-way interaction between age group, cue validity, and $\mathrm{SOA}, F(6,351)=2.97$, $p<.01$. Validity effects, when examined at each SOA with simple effects testing, reflected significant facilitation for all three age groups at the 100- and 300-ms SOAs, all $F_{\mathrm{S}}>5.0$, all $p \mathrm{~s}<.05$. Group differences in the magnitude of facilitation at these SOAs (Fig. 4a), as assessed with difference scores (invalid RT minus valid RT), were significant at the 300-ms SOA, $F(2,117)=4.02, p<.05$; young-old adults and old-old adults had significantly greater facilitation effects than did young adults, $p \mathrm{~s}<.05$.

Following Brinley transformation of the data for young and young-old participants, using Eqs. 5 and 6, the main effect of age from the overall ANOVA was no longer significant, $F<1$, as expected. The other effects from the original analysis remained significant, including the three-way interaction of age group, cue validity, and SOA, $F(6,351)=2.27, p<.05$. However, when age differences in facilitation effects were explored at each SOA, the age difference at the $300-\mathrm{ms}$ SOA was now marginally significant, $F(2,117)=2.64, p=.08$.

Old-Old RT $=1.28($ Young RT $)+64, r^{2}=.88$

Old-Old RT $=0.97($ Young-Old RT $)+67, r^{2}=.97$

Peripheral cues For peripheral cues, two main effects were significant: age group, $F(2,117)=48.22, p<.0001$, and SOA, $F(3,351)=205.05, p<.0001$. There were two significant two-way interactions: Age Group $\times$ Cue Validity, $F(2,117)=8.16, p<.001$, and Cue Validity $\times$ SOA, $F(3,351)=75.84, p<.0001$, which were qualified by a three-way interaction between age group, cue validity, and SOA, $F(6,351)=2.46, p<.05$. Simple main effects testing assessed the validity effects for each age group at each SOA. Facilitation effects were significant for all three age groups at the 100-ms SOA, all $F_{\mathrm{S}}>16.5$, all $p \mathrm{~s}<.001$. Only the old-old group continued to show facilitation at the 300-ms SOA, $F(1,39)=15.12, p<.001$. At 600 and $1,000 \mathrm{~ms}$, IOR effects were significant for young adults and for young-old adults, all $F \mathrm{~s}>17.0$, all $p \mathrm{~s}<.001$. The IOR effects for old-old adults were significant at $1,000 \mathrm{~ms}$, $F(1,39)=13.12, p<.001$, but not at $600 \mathrm{~ms}, F<1$. Group differences in validity effects, as assessed with difference 
Table 3 Mean reaction times in milliseconds (and standard deviations) for Experiment 2

\begin{tabular}{|c|c|c|c|c|c|c|c|c|}
\hline \multirow[b]{2}{*}{ SOA (ms) } & \multicolumn{4}{|c|}{ Arrow Cues } & \multicolumn{4}{|c|}{ Peripheral Cues } \\
\hline & 100 & 300 & 600 & 1,000 & 100 & 300 & 600 & 1,000 \\
\hline \multicolumn{9}{|l|}{ Young } \\
\hline Valid & $364(56)$ & $326(60)$ & $321(53)$ & $311(52)$ & $380(52)$ & $357(60)$ & $359(62)$ & $341(57)$ \\
\hline Invalid & $387(58)$ & $333(65)$ & $325(60)$ & $316(60)$ & $400(59)$ & $357(61)$ & $334(59)$ & $320(58)$ \\
\hline \multicolumn{9}{|l|}{ Young-old } \\
\hline Valid & $471(87)$ & $440(83)$ & $412(76)$ & $414(75)$ & 497 (72) & $451(67)$ & $457(81)$ & $444(74)$ \\
\hline Invalid & $504(87)$ & $463(81)$ & 407 (72) & $405(73)$ & $521(82)$ & $460(76)$ & $428(82)$ & 414 (77) \\
\hline \multicolumn{9}{|l|}{ Old-old } \\
\hline Valid & $528(105)$ & $493(90)$ & $470(90)$ & 462 (107) & $542(104)$ & $500(91)$ & $499(90)$ & $505(100)$ \\
\hline Invalid & $556(96)$ & $516(87)$ & 474 (99) & 454 (102) & $594(113)$ & $530(101)$ & 494 (102) & 481 (94) \\
\hline
\end{tabular}

scores (invalid RT minus valid RT), were significant at SOAs of $100 \mathrm{~ms}, F(2,117)=10.39, p<.0001,300 \mathrm{~ms}$, $F(2,117)=5.10, p<.01$, and $600 \mathrm{~ms}, F(2,117)=4.30$, $p<.05$. Old-old adults had significantly greater facilitation (or, at $600 \mathrm{~ms}$, significantly less inhibition) than did young-old

(a) Arrow Cues

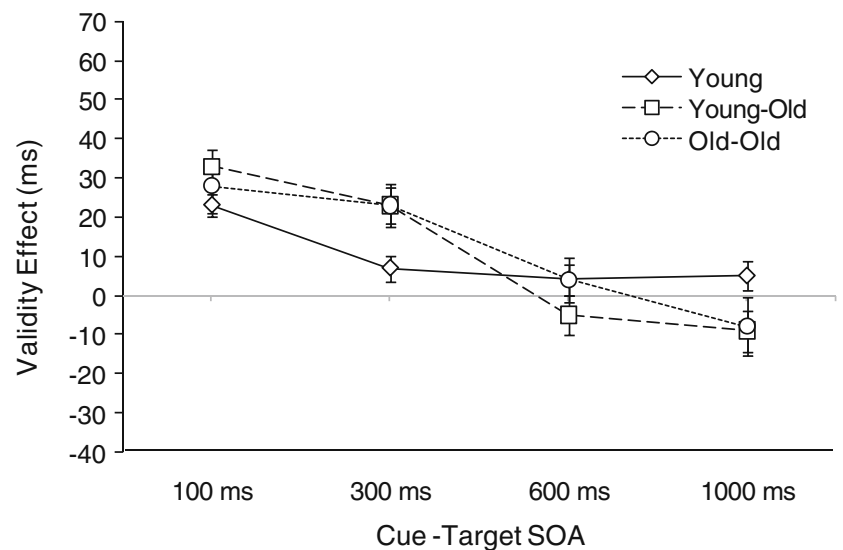

(b) Peripheral Cues

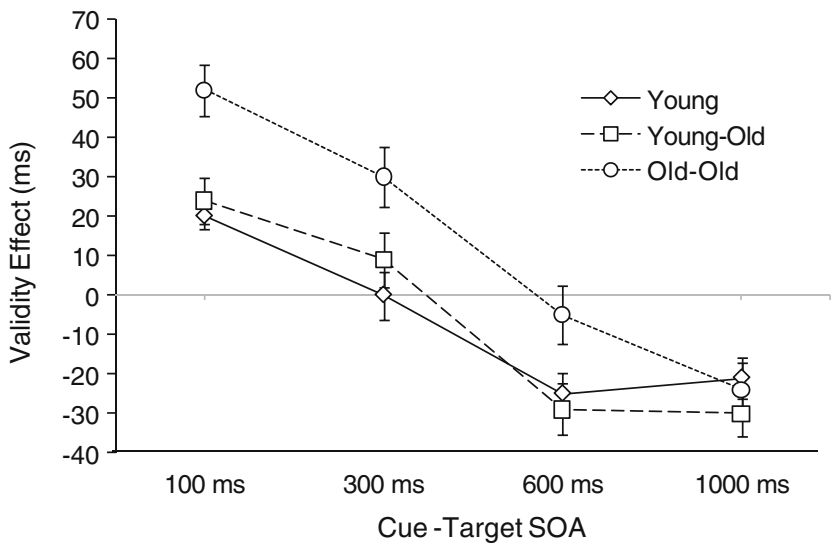

Fig. 4 Validity effects (difference scores: invalid RT minus valid RT) as a function of cue-target SOA for young, young-old, and old-old adults in Experiment 2. a Uninformative central arrow cues. b Uninformative peripheral onset cues. Error bars represent $\pm 1 S E M$ and young adults, $p \mathrm{~s}<.05$. Difference scores reflecting validity effects are presented in Fig. 4b.

Following a Brinley transformation using Eqs. 7 and 8, the main effect of group from the overall ANOVA was no longer significant, $F<1$. The two-way interactions between age group and cue validity, $F(2,117)=7.95, p<.001$, and between cue validity and SOA, $F(3,351)=75.99$, $p<.0001$, did continue to be significant, but the threeway interaction was no longer significant, $F(6,351)=1.47$, $p>$.15. All other significance patterns remained unchanged from the original analysis, including significant group differences in validity effects at the 100-, 300-, and 600-ms SOAs, all $F \mathrm{~s}>4.4$, all $p \mathrm{~s}<.05$, with old-old adults having greater facilitation effects (or, at $600 \mathrm{~ms}$, smaller inhibition effects) than the other two age groups.

Old-Old RT $=1.31($ Young RT $)+53, r^{2}=.83$

Old-Old RT $=0.98($ Young-Old RT $)+67, r^{2}=.89$

Errors For both arrow and peripheral cues, error rates were low across all conditions for each age group (ranging between $0.1 \%$ and $2.4 \%$ ), so no further error analyses were conducted.

\section{Discussion}

The time course of spatial orienting for young adults was relatively constant across the two experiments. Orienting was facilitated by valid arrow cues at the shortest cue-target interval (100-ms SOA), and this facilitation effect diminished at longer intervals. With peripheral cues, young adults' responses reflected facilitation at the shortest interval (100-ms SOA) and inhibition at the longer intervals (600- and 1,000-ms SOAs). Thus, overall, the observed orienting pattern for young adults was relatively unaffected 
by cue duration, although a casual comparison of Figs. 2 and 4 suggests that young adults' IOR effects were greater in magnitude following brief peripheral cues (Exp. 2) than following persistent cues (Exp. 1).

Removing the cue prior to target presentation was effective in modifying age-related patterns of orienting, but more markedly so for peripheral cues. For arrow cues, as in Experiment 1, facilitation effects were greater in magnitude for the two older groups than for young adults at the 300-ms SOA, although the age difference did not survive the Brinley transformation. Thus, from a conservative standpoint, a brief arrow cue led to less-pronounced age differences in facilitation effects than did a persistent cue. Both older groups demonstrated IOR when the peripheral cue was removed prior to target presentation (Exp. 2), but not when the cue remained present (Exp. 1), With a brief cue, the young-old group's validity effects were now virtually identical to those of young adults (facilitation at the 100-ms SOA and IOR at the 600- and 1,000-ms SOAs, with no differences between the two groups in the magnitude of the effects). The old-old group's performance did not benefit as much from a shortened cue. They continued to show greater facilitation than did the other two age groups at the shorter SOAs (100 and $300 \mathrm{~ms}$ ) and a delayed development of IOR at the longer SOAs (with IOR at 1,000 ms but not at $600 \mathrm{~ms}$ ).

In accord with the predictions of ACS theory, age differences in orienting performance were still evident in Experiment 2. With a short-duration arrow cue, both older groups showed greater facilitation effects than did young adults, suggesting slower-resolving orienting effects, although general slowing likely did contribute to this age difference. For peripheral cues, enhanced facilitation effects were limited to the old-old group. Both older groups showed IOR, although the onset of IOR was delayed for old-old adults. Although older adults may have set attentional controls to higher settings due to greater perceived task difficulty, these settings appeared to have had less of an impact on orienting to peripheral cues when the cue was removed prior to target presentation. Removing the cue likely facilitated endogenous disengagement of attention from the cued location (and thus facilitated IOR), perhaps more so for young-old adults (whose performance became similar to that of young adults) than for old-old adults.

\section{General discussion}

Reflexive orienting initiated by central arrow cues and peripheral onset cues

We examined the time course of reflexive orienting to two types of visuospatial cues, central arrow cues and peripheral onset cues. The cues did not reliably predict the location of an upcoming target, yet young adults and older adults reliably shifted attention based on the location information provided by the cues. The early time point at which cues influenced orienting ( $100 \mathrm{~ms}$ between the onsets of the cues and targets) indicated that attentional orienting was reflexively guided. Facilitation effects (faster responses to validly than to invalidly cued targets) at short cue-target intervals were replaced by IOR (slower responses to validly than to invalidly cued targets) at longer cue-target intervals, but only following peripheral onset cues, consistent with the idea that peripheral cues uniquely activated components of the eye movement system (e.g., frontal eye fields, superior colliculus) that are associated with attentional inhibition (Lepsien \& Pollman, 2002; Posner, Rafal, Choate, \& Vaughan, 1985; Sapir, Soroker, Berger, \& Henik, 1999). The facilitation effects resulting from arrow cues were not followed by inhibition effects, and these facilitation effects were largely resolved by $600 \mathrm{~ms}$.

How do central symbolic cues assume reflexive orienting properties? In the case of the arrow, it is a symbol that is pervasively used in our daily lives to indicate the locations of objects or destinations (e.g., traffic exits, turn lanes, airport gates, stores in the mall). Arrows in these contexts are highly predictive of desired locations. Thus, with accumulating experience, observers may come to automatically orient in the direction indicated by an arrow. Another possibility is that orienting in response to the directional information provided by arrows is not learned but occurs naturally, because arrows are inherently directional (Freyd \& Pantzer, 1995). This notion is consistent with the results of a study by Ristic et al. (2002), in which 4- and 5-year-old children oriented in response to uninformative arrow cues, despite their limited experience with the predictive properties of arrows in everyday life. In either case, the findings from the present study suggest that automatic orienting in response to arrows does not diminish with age, even in advanced old age. In fact, it appears that the effect of arrow cues on attentional orienting is actually enhanced later in life.

Age differences in reflexive orienting to peripheral onset cues

We found that age and cue duration interacted to influence the temporal patterns of orienting to peripheral onset cues. With a persistent cue (Exp. 1), both young-old and old-old adults showed enhanced facilitation effects relative to young adults. With a brief cue (Exp. 2), only old-old adults continued to show enhanced facilitation effects. Cue duration impacted on IOR effects as well. Young adults demonstrated IOR toward peripheral cues that remained on the screen (Exp. 1), but older adults did not. In contrast, when peripheral cues were removed prior to target onset 
(Exp. 2), all three age groups demonstrated IOR, although IOR onset was delayed for old-old adults. Thus, the facilitation and IOR patterns suggest that reducing cue duration was successful in prompting cue disengagement for older adults, particularly for young-old adults.

The cuing patterns above indicate that peripheral cues were effective in capturing older adults' attention. In fact, older adults may have been particularly prone to automatic capture. The age-related increase in the magnitude and duration of facilitation effects, consistent with findings from other studies (Brodeur \& Enns, 1997; Castel et al., 2003; Faust \& Balota, 1997; Lincourt et al., 1997), likely reflected older adults' stronger engagement with and delayed disengagement from peripheral cues. In addition, the extended duration of cue engagement may have interfered with the expression of IOR (Klein, 2000, 2005). When a peripheral cue remained visible over the cue-target interval, thus promoting continued engagement at the cued location, older adults failed to demonstrate IOR (Exp. 1). In contrast, when the cue was removed prior to the target's appearance, older adults' endogenous disengagement from the cue was facilitated, and consequently IOR to the cued location was revealed (Exp. 2), although IOR onset was still delayed for old-old adults. In studies using a cue-back IOR task (in which a second central cue is used to draw attention back to fixation), older adults show no delay in the development of IOR (Faust \& Balota, 1997; Langley, Fuentes, Vivas, \& Saville, 2007), reinforcing the idea that if conditions are conducive for older adults to remove attention from a cued location, IOR will emerge for this group. Young adults, on the other hand, appear able to efficiently remove attention from the cue, even when the cue remains physically present (thus leading to IOR in Exp. 1), suggesting greater flexibility in attentional shifting for this age group, and the least flexibility in shifting performance for old-old adults.

The IOR pattern of the present study differs from that identified by Castel et al. (2003). Using a brief-duration cue (50 ms, as in Exp. 2 of the present study), Castel and colleagues found that IOR onset was observed in young adults at a cue-target SOA of $250 \mathrm{~ms}$, but was delayed for older adults until an SOA of $750 \mathrm{~ms}$. With a brief cue (Exp. 2), we found a delay in IOR onset only for old-old adults. One difference between these studies is the time point at which IOR was first observed in young adults (IOR emerged at a $600-\mathrm{ms} \mathrm{SOA}$ in the present study but at a 250 ms SOA in the Castel et al. study). Differences in the target task may account for these between-study differences in IOR onset; we used a discrimination task (indicating the left or right location of the target), whereas Castel et al. used a detection task (pressing a button as soon as the target was detected). The onset of IOR is known to lag in discrimination tasks relative to detection tasks (Lupiáñez, Milán, Tornay, Madrid, \& Tudela, 1997). However, task differences cannot explain the difference in the age patterns of IOR between the studies. Castel et al. found age differences at $250 \mathrm{~ms}$ (young adults showed IOR, whereas older adults showed facilitation) and at $500 \mathrm{~ms}$ (older adults showed less IOR than did younger adults). In contrast, we found no age differences in IOR between the young-old and young groups when IOR was first observed at $600 \mathrm{~ms}$. Consistent with our findings (at SOAs of 600 and 1,000 ms), both age groups in Castel et al. showed IOR at 750 and 1,000 ms. It is possible that we would have observed age differences in the onset of IOR (with young adults developing IOR sooner than young-old adults) in Experiment 2 if we had sampled SOAs between 300 and $600 \mathrm{~ms}$. Nevertheless, it is still the case that we found an impact of cue duration on observed age differences in IOR, with age differences greatly reduced (older adults now showing IOR, although it was still delayed in old-old adults) when the cue was removed prior to target presentation.

The pattern of results with peripheral cues was largely consistent with Klein's (2005) ACS theory of attentional orienting. It is important to note that, based on the findings from the Brinley plot transforms, the observed age differences in the facilitation and IOR effects were not completely accounted for by general slowing on the part of older adults (Faust et al., 1999). This leaves open the possibility that attention-specific accounts, such as the ACS theory, could explain the observed age patterns. In accord with the ACS theory, we interpreted the results as indicating that older adults used a higher control setting than did younger adults because they found the location discrimination task more difficult. The higher setting heightened the intensity of attention to the cue as well as to the target, leading cueinduced orienting to be associated with enhanced facilitation and delayed inhibition. The control setting's influence on older adults' reflexive orienting was most evident for persistent cues, which, at the cued location, would have been particularly difficult to disengage from.

We found evidence that old-old adults used a higher attentional control setting than did young-old adults, but only with brief cues. With persistent cues, the two older groups oriented to peripheral cues in similar manners (with little difference in the magnitudes and time courses of validity effects). With brief peripheral cues (Exp. 2), old-old adults showed greater-magnitude facilitation at short cue-target intervals than did young-old adults, as well as later-developing IOR. This pattern suggested that cue offset was less effective in encouraging old-old adults' early endogenous disengagement from the cued location. However, old-old adults did demonstrate IOR in Experiment 2, indicating improved cue disengagement relative to Experiment 1 . The enhanced early facilitation effect for old-old adults is consistent with Greenwood and Parasuraman's (1994) finding that old-old adults had greater cuing effects than did young-old adults when 
orienting to informative peripheral arrows. Because we used uninformative peripheral onset cues, we can be more certain that our results reflected changes in reflexive orienting (as opposed to some combination of reflexive and volitional orienting). Together, the findings suggest that the control setting used by old-old adults was at the same level, or perhaps slightly higher, than the setting used by young-old adults. In addition, cue offset was not as effective in this group at discouraging prolonged attentional engagement. It is worth investigating age differences for an orienting task requiring a detection response; it is possible that had we used a simpler task (thus lowering the control setting), age differences in orienting would have diminished.

Faust and Balota (1997) found that older adults showed greater facilitation effects than did young adults (tested at a cue-target SOA of $200 \mathrm{~ms}$ ), and they proposed that this difference was due to a breakdown in the posterior attention system with age, which led to a reduced ability to localize objects in the visual field. As a result, older adults benefited more than young adults from an onset cue that drew attention to a location. A reduced ability to localize objects could account for the present age differences in the benefit of a valid location cue, but it could not explain why there would be age differences in the time courses of cuing effects (reflecting delayed disengagement from cues, particularly when the cues remained physically present). The ACS theory provides an alternative explanation for age-related increases in facilitation effects that also addresses changes in the time course of cue validity effects. According to this theory, age-related increases in task difficulty influenced the attentional control settings applied to the cues as well as to the targets. As a result of higher control settings, older adults produced enhanced and prolonged facilitation effects and delayed inhibition effects.

What the ACS theory does not specify is what makes the target-processing task more difficult for older adults than for young adults, thus leading to higher control settings. The present study did not address the source of the task difficulty, but there are at least three possibilities. First, agerelated changes in central or peripheral acuity may have influenced how quickly targets in the periphery were detected and processed (Schneider \& Pichora-Fuller, 2000). Although we limited the sample to individuals with visual acuities of 20/40 or better, age differences in central acuity were observed and may have influenced target processing, and changes in peripheral acuity (not assessed) may have influenced initial detection. Second, age-related general slowing (evidenced in the present study in terms of age-related increases in overall RTs) may have delayed or hampered target processing for older adults (Kramer \& Madden, 2008). Higher control settings associated with orienting to cues and targets may have compensated for this slowing in stimulus processing. Finally, age-related changes specific to response selection in a forced choice task may have influenced task difficulty, above and beyond the general slowing. That said, similar age patterns in orienting effects (enhanced and prolonged facilitation effects for older adults) have been found with single-choice detection tasks, suggesting that age differences in task difficulty are still found on tasks that are less demanding of response selection processes (see, e.g., Castel et al., 2003). Future studies will need to delineate the age-related factors that influence task difficulty in orienting tasks.

Age differences in reflexive orienting to central arrow cues

In the present study, young adults and older adults showed similar orienting patterns to uninformative central arrow cues. Responses were facilitated by valid relative to invalid cues at the shortest cue-target interval (100-ms SOA), indicating reflexive and rapid orienting of attention. Facilitation effects were resolved by $300 \mathrm{~ms}$ for young adults, and by $600 \mathrm{~ms}$ for young-old and old-old adults. This relatively brief influence on orienting is in contrast to the sustained facilitation effects typically found with informative central arrows (Olk, Cameron, \& Kingstone, 2008; Ristic \& Kingstone, 2006) and suggests that our participants did not have the same incentive (in terms of likely target appearance) to maintain attention at the cued location. In Experiment 1 and Experiment 2, the facilitation effects for older adults were maintained at $300 \mathrm{~ms}$, whereas these effects for young adults had diminished by the same time point. In contrast to the pattern observed with peripheral cues, cue duration had less of an impact on age differences in orienting to central arrows. When cue duration was shortened, both older groups continued to have greater facilitation effects than did young adults (although not after controlling for general slowing).

Applying the ACS theory to central arrow cues is not as straightforward as applying it to peripheral onset cues. Do the control settings affect engagement and disengagement with the arrow cue itself, or with the location indicated by the cue? Because a peripheral cue occurs at the cued location, engagement with and disengagement from a cue also reflects engagement with and disengagement from the cued location. However, in the case of a central arrow, greater engagement with the cue itself would mean greater attention at the center location. If the result of a higher setting is greater attention to the location indicated by the cue (left location for a left arrow, right location for a right arrow), then facilitation effects would be enhanced. The finding that central arrow cues led to enhanced cuing effects for older adults relative to young adults suggests that older adults had greater engagement with the cued location, rather than with the cue itself. Thus, if one accepts that attention control settings impacted how strongly 
observers reacted to the directional information provided by the cue rather than how strongly attention was directed to the location of the cue, ACS theory can explain orienting in response to both arrow and peripheral cues. This interpretation may explain why cue duration had relatively little effect on orienting to arrow cues. Once attention had been shifted to the cued location, based on the arrow information, there was no need to disengage from the arrow cue itself, even when it remained present. Together, there is mounting evidence that the ACS theory can account for age patterns in reflexive orienting to both central arrows and peripheral onset cues.

Central arrow cues in past aging studies have consistently been informative (e.g., Folk \& Hoyer, 1992; Lincourt et al., 1997; Tellinghuisen et al., 1996; Yamaguchi et al., 1995), and orienting to such cues was thought to reflect volitional shifts of attention. In these past studies, both young adults and older adults demonstrated reliable facilitation effects, and in some cases, the facilitation effects were greater in magnitude for older adults (Curran et al., 2001; Hartley et al., 1990; Lincourt et al., 1997; Nissen \& Corkin, 1985). This pattern was interpreted as indicating that volitional orienting remained intact with age. Future studies will need to disentangle the contributions of volitional and reflexive orienting to older adults' responses to predictive central arrow cues. Recent work has indicated that informative arrows trigger an interaction between volitional and reflexive orienting that produces orienting effects that exceed the predicted additive combination of the individual orienting processes (Olk et al., 2008; Ristic $\&$ Kingstone, 2006). Age differences in either reflexive or volitional orienting alone, or age differences in both of these processes, may change how the processes interact to produce orienting to predictive central arrows.

\section{Implications}

The question remains whether age differences in orienting represent an adaptive or maladaptive change for older adults. The answer depends in part on how we use peripheral onset and arrow cues in real-world contexts (Kingstone et al., 2003). It is easy to imagine contexts in which we encounter orienting cues-driving, navigating unfamiliar environments (e.g., the airport, the mall, an amusement park), or searching for information on Internet sites. For example, Internet advertisers have discovered that advertisements that involve dynamic visual displays (which function as peripheral cues) are particularly difficult to ignore (due to reflexive shifts of attention). In addition, arrows presented on Internet sites direct us to further information and links (and these arrows are likely to be both reflexively and volitionally attended). In real-life situations, how will age-related changes in the magnitude and timing of orienting influence the success of such cues in guiding attention? In the lab, enhanced orienting toward valid cues can help older adults more rapidly localize sought-after information, which certainly could be adaptive in real-life situations, particularly given an age-related slowing in cognitive processing. However, more intense processing of invalid cues hinders attentional shifts to desired target locations, and slower cue disengagement delays IOR to previously searched locations. These alterations have implications for spatial orienting in the real world, particularly in situations in which misleading visual cues automatically capture older adults' attention but do not predict the location of desired information. Delayed disengagement may slow older adults' visual search in real-world contexts and cause them to prematurely return attention to searched locations. Thus, the adaptiveness of these age-related changes in orienting may depend on the visual context and the predictability of visual cues.

Author Note This work was supported by Centers of Biomedical Research Excellence (COBRE) Grant P20 RR020151 from the National Center for Research Resources (NCRR), a component of the National Institutes of Health (NIH). The contents of this report are solely the responsibility of the authors and do not necessarily reflect the official views of the NIH or NCRR. We are grateful to Ganesh Padmanabhan for programming the experiments and to Shanna Morlock, Jaryn Allen, Laura Klubben, Savannah Kraft, Melissa Tarasenko, Lindsay Anderson, Heather Wadeson, Heather Joyce, Tanya Peterson, RaeAnn Levang, Veselin Marinov, Joseph Gabel, and Nicole Kiewel for collecting the data.

\section{References}

Abrams, R. A., \& Dobkin, R. S. (1994). Inhibition of return: Effects of attentional cuing on eye movement latencies. Journal of Experimental Psychology: Human Perception and Performance, 20, 467-477. doi:10.1037/0096-1523.20.3.467

Brodeur, D. A., \& Enns, J. T. (1997). Covert visual orienting across the lifespan. Canadian Journal of Experimental Psychology, 51, 20-35. doi:10.1037/1196-1961.51.1.20

Castel, A. D., Chasteen, A. L., Scialfa, C. T., \& Pratt, J. (2003). Adult age differences in the time course of inhibition of return. Journal of Gerontology: Psychological Sciences, 58B, P256-P259.

Cerella, J. (1994). Generalized slowing in Brinley plots. Journal of Gerontology: Psychological Sciences, 49B, P65-P71.

Christensen, K. J., Moye, J., Armson, R. R., \& Kern, T. M. (1992). Health screening and random recruitment for cognitive aging research. Psychology and Aging, 7, 204-208. doi:10.1037/08827974.7.2.204

Curran, T., Hills, A., Patterson, M. B., \& Strauss, M. E. (2001). Effects of aging on visuospatial attention: An ERP study. Neuropsychologia, 39, 288-301. doi:10.1016/S0028-3932(00) 00112-3

Faust, M. E., \& Balota, D. A. (1997). Inhibition of return and visuospatial attention in healthy older adults and individual with dementia of the Alzheimer type. Neuropsychology, 11, 13-29. doi:10.1037/0894-4105.11.1.13

Faust, M. E., Balota, D. A., Spieler, D. H., \& Ferraro, F. R. (1999). Individual differences in information-processing rate and amount: Implications for group differences in response 
latency. Psychological Bulletin, 125, 777-799. doi:10.1037/ 0033-2909.125.6.777

Folk, C. L., \& Hoyer, W. J. (1992). Aging and shifts of visual spatial attention. Psychology and Aging, 7, 453-465. doi:10.1037/08827974.7.3.453

Folk, C. L., Remington, R., \& Johnston, J. C. (1992). Involuntary covert orienting is contingent on attentional control settings. Journal of Experimental Psychology: Human Perception and Performance, 18, 1030-1044. doi:10.1037/0096-1523.18.4.1030

Folstein, M. F., Folstein, S. E., \& McHugh, P. R. (1975). Mini-mental state: A practical method for grading the cognitive state of the patient for the clinician. Journal of Psychiatric Research, 12, 189-198. doi:10.1016/0022-3956(75)90026-6

Freyd, J. J., \& Pantzer, T. M. (1995). Static patterns moving in the mind. In S. M. Smith, T. B. Ward, \& R. A. Finke (Eds.), The creative cognition approach (pp. 181-204). Cambridge, MA: MIT Press.

Friesen, C. K., \& Kingstone, A. (1998). The eyes have it! Reflexive orienting is triggered by nonpredictive gaze. Psychonomic Bulletin \& Review, 5, 490-495.

Greenwood, P. M., \& Parasuraman, R. (1994). Attentional disengagement deficit in nondemented elderly over 75 years of age. Aging and Cognition, 1, 188-202. doi:10.1080/13825589408256576

Greenwood, P. M., Parasuraman, R., \& Haxby, J. V. (1993). Changes in visuospatial attention over the adult lifespan. Neuropsychologia, 31, 471-485. doi:10.1016/0028-3932(93)90061-4

Hartley, A. A., \& Kieley, J. M. (1995). Adult age differences in the inhibition of return of visual attention. Psychology and Aging, 10, 670-683. doi:10.1037/0882-7974.10.4.670

Hartley, A. A., Kieley, J. M., \& Slabach, E. H. (1990). Age differences and similarities in the effects of cues and prompts. Journal of Experimental Psychology: Human Perception and Performance, 16, 523-537. doi:10.1037/0096-1523.16.3.523

Hommel, B., Pratt, J., Colzato, L., \& Godijn, R. (2001). Symbolic control of visual attention. Psychological Science, 12, 360-365. doi:10.1111/1467-9280.00367

Huey, E. D., \& Wexler, B. E. (1994). Abnormalities in rapid, automatic aspects of attention in schizophrenia: Blunted inhibition of return. Schizophrenia Research, 14, 57-63. doi:10.1016/ 0920-9964(94)90009-4

Jonides, J. (1981). Voluntary versus automatic control over the mind's eye's movement. In J. Long \& A. Baddeley (Eds.), Attention and performance $I X$ (pp. 187-203). Hillsdale, NJ: Erlbaum.

Kingstone, A., Smilek, D., Ristic, J., Friesen, C. K., \& Eastwood, J. D. (2003). Attention, researchers! It is time to take a look at the real world. Current Directions in Psychological Science, 12, 176180. doi:10.1111/1467-8721.01255

Klein, R. M. (2000). Inhibition of return. Trends in Cognitive Sciences, 4, 138-147. doi:10.1016/B978-012375731-9/50020-3

Klein, R. M. (2005). On the role of endogenous orienting in the inhibitory aftermath of exogenous orienting. In U. Mayr, E. Awh, \& S. W. Keele (Eds.), Developing individuality in the human brain: $A$ tribute to Michael I. Posner (pp. 45-64). Washington DC: American Psychological Association. doi:10.1037/11108-003

Klein, R. M., Kingstone, A., \& Pontefract, A. (1992). Orienting of visual attention. In K. Rayner (Ed.), Eye movements and visual cognition: Scene perception and reading (pp. 46-63). Amsterdam: Elsevier Science.

Kramer, A. F., \& Madden, D. J. (2008). Attention. In F. I. M. Craik \& T. A. Salthouse (Eds.), The handbook of aging and cognition (3rd ed., pp. 189-249). New York: Psychology Press.

Langley, L. K., Fuentes, L. J., Vivas, A. B., \& Saville, A. L. (2007). Aging and temporal patterns of inhibition of return. Journal of Gerontology: Psychological Sciences, 62B, P71-P77.

Lepsien, J., \& Pollman, S. (2002). Covert reorienting and inhibition of return: An event-related fMRI study. Journal of Cognitive Neuroscience, 14, 127-144. doi:10.1162/089892902317236795
Lincourt, A. E., Folk, C. L., \& Hoyer, W. J. (1997). Effects of aging on voluntary and involuntary shifts of attention. Aging, Neuropsychology, and Cognition, 4, 290-303. doi:10.1080/ 13825589708256654

Lupiáñez, J., Milán, E. G., Tornay, F. J., Madrid, E., \& Tudela, P. (1997). Does IOR occur in discrimination tasks? Yes, it does, but later. Perception \& Psychophysics, 59, 1241-1254.

Lupiáñez, J., Milliken, B., Solano, C., Weaver, B., \& Tipper, S. P. (2001). On the strategic modulation of the time course of facilitation and inhibition of return. The Quarterly Journal of Experimental Psychology, 54A, 753-773. doi:10.1080/ 02724980042000453

MacPherson, A. C., Klein, R. M., \& Moore, C. M. (2003). Inhibition of return in children and adolescents. Journal of Experimental Child Psychology, 85, 337-351. doi:10.1016/S0022-0965(03)00104-8.

Madden, D. J., Pierce, T. W., \& Allen, P. A. (1992). Adult age differences in attentional allocation during memory search. Psychology and Aging, 7, 594-601. doi:10.1037/08827974.7.4.594

Madden, D. J., Whiting, W. L., Cabeza, R., \& Huettel, S. A. (2004). Age-related preservation of top-down attentional guidance during visual search. Psychology and Aging, 19, 304-309. doi:10.1037/ 0882-7974.19.2.304

Nissen, M. J., \& Corkin, S. (1985). Effectiveness of attentional cueing in older and younger adults. Journal of Gerontology, 40, 185-191.

Olk, B., Cameron, B., \& Kingstone, A. (2008). Enhanced orienting effects: Evidence for an interaction principle. Visual Cognition, 16, 979-1000. doi:10.1080/13506280701848921

Posner, M. I., \& Cohen, Y. A. (1984). Components of visual orienting. In H. Bouma \& D. G. Bouwhuis (Eds.), Attention and performance X: Control of language processes (pp. 531-554). Hillsdale, NJ: Erlbaum.

Posner, M. I., Rafal, R. D., Choate, L. S., \& Vaughan, J. (1985). Inhibition of return: Neural basis and function. Journal of Cognitive Neuropsychology, 2, 211-228. doi:10.1080/ 02643298508252866

Rafal, R. D., Calabresi, P. A., Brennan, C. W., \& Sciolto, T. K. (1989). Saccade preparation inhibits reorienting to recently attended locations. Journal of Experimental Psychology: Human Perception and Performance, 15, 673-685. doi:10.1037/0096-1523.15.4.673

Ristic, J., Friesen, C. K., \& Kingstone, A. (2002). Are eyes special? It depends on how you look at it. Psychonomic Bulletin \& Review, 9, 507-513.

Ristic, J., \& Kingstone, A. (2006). Attention to arrows: Pointing to a new direction. The Quarterly Journal of Experimental Psychology, 59, 1921-1930. doi:10.1080/17470210500416367

Sapir, A., Henik, A., Dobrusin, M., \& Hochman, E. Y. (2001). Attentional asymmetry in schizophrenia: Disengagement and inhibition of return deficits. Neuropsychology, 5, 361-370. doi:10.1037/0894-4105.15.3.361

Sapir, A., Soroker, N., Berger, A., \& Henik, A. (1999). Inhibition of return in spatial attention: Direct evidence for collicular generation. Nature Neuroscience, 2, 1053-1054. doi:10.1038/15977

Schneider, B. A., \& Pichora-Fuller, M. K. (2000). Implications of perceptual deterioration for cognitive aging research. In F. I. M. Craik \& T. A. Salthouse (Eds.), The handbook of aging and cognition (2nd ed., pp. 155-219). Mahwah, NJ: Erlbaum.

Spencer, K. M., Nestor, P. G., Valdman, O., Niznikiewicz, M. A., Shenton, M. E., \& McCarley, R. W. (2011). Enhanced facilitation of spatial attention in schizophrenia. Neuropsychology, 25, 7685. doi:10.1037/a0020779

Tales, A., Muir, J. L., Bayer, A., \& Snowden, R. J. (2002). Spatial shifts in visual attention in normal ageing and dementia of the Alzheimer type. Neuropsychologia, 40, 2000-2012. doi:10.1016/ S0028-3932(02)00057-X 
Tellinghuisen, D. J., Zimba, L. D., \& Robin, D. A. (1996). Endogenous visuospatial precuing effects as a function of age and task demands. Perception \& Psychophysics, 58, 947-958.

Tipples, J. (2002). Eye gaze is not unique: Automatic orienting in response to uninformative arrows. Psychonomic Bulletin \& Review, 9, 314-318. doi:10.3758/BF03196287

Wechsler, D. (1999). Wechsler Abbreviated Scale of Intelligence. San Antonio, TX: Psychological Corp.
Yamaguchi, S., Tsuchiya, H., \& Kobayashi, S. (1995). Electrophysiologic correlates of age effects on visuospatial attention shift. Cognitive Brain Research, 3, 41-49. doi:10.1016/0926-6410(95)00017-8

Yesavage, J. A., Brink, T. L., Rose, T. L., Lum, O., Huang, V., Adey, M., et al. (1982). Development and validation of a geriatric depression screening scale: A preliminary report. Journal of Psychiatric Research, 17, 37-49. doi:10.1016/0022-3956(82) 90033-4 